\title{
Regards sur les médias dans le conflit Yougoslave
}

Alice Krieg

\section{OpenEdition}

Journals

Édition électronique

URL : https://journals.openedition.org/questionsdecommunication/6469

DOI : 10.4000/questionsdecommunication.6469

ISSN : 2259-8901

\section{Éditeur}

Presses universitaires de Lorraine

\section{Édition imprimée}

Date de publication : 1 mars 2002

ISBN : 978-2-86480-839-8

ISSN : 1633-5961

\section{Référence électronique}

Alice Krieg, «Regards sur les médias dans le conflit Yougoslave », Questions de communication [En

ligne], 1 | 2002, mis en ligne le 01 mars 2002, consulté le 09 décembre 2021. URL : http:// journals.openedition.org/questionsdecommunication/6469; DOI : https://doi.org/10.4000/ questionsdecommunication.6469

Ce document a été généré automatiquement le 9 décembre 2021. 


\title{
Regards sur les médias dans le conflit Yougoslave
}

\author{
Alice Krieg
}

1 La couverture des guerres yougoslaves par les médias a suscité, à l'instar de celle d'autres événements internationaux de la fin $\mathrm{du} 20^{\mathrm{e}}$ siècle (révolution roumaine, intervention américaine en Somalie, guerre du Golfe, génocide au Rwanda...), travaux et réflexions de la part de différentes catégories d'acteurs et de témoins. Inégaux tant dans leurs visées que dans l'ampleur de travail requis ou encore dans leur visibilité dans l'espace public, tous apportent un éclairage sur le conflit yougoslave et sur la façon dont les médias en ont organisé le récit.

2 Nous proposons ici un panorama des différents «travaux » - terme général qui inclut les essais, les pamphlets, etc. - qui ont pu être proposés sur le sujet «médias et guerres yougoslaves ». Sur quelle(s) guerre(s) yougoslave(s) les auteurs se sont-ils penchés ? Quel type de point de vue ont-ils adopté ? Quels médias ont-ils privilégiés ? Les auteurs se sont-ils intéressés aux contenus, aux formes, aux pratiques? Notre état des lieux des regards critiques portés sur le thème « médias et guerres yougoslaves » suggère que la question de l'influence des médias sur la construction de l'opinion est en définitive une des motivations fortes des auteurs, que ceux-ci appartiennent ou non à la sphère scientifique.

\section{Typologie des travaux}

3 Un premier critère de différenciation des travaux existants est celui de la guerre donc de la période - concernée. Selon le moment où leur désir de travail s'est fait jour, selon les demandes sociales ressenties, selon les facilités pratiques qui s'offraient, et peut-être selon la perspective politique qu'ils entendaient défendre, les « auteurs »terme général, lui aussi - ont fait porter leur travail plutôt sur la première série des guerres yougoslaves (Slovénie, Croatie, Bosnie, 1991-1995) ou plutôt sur la seconde série (Kosovo, 1998-1999), mais rarement sur l'ensemble des conflits (dont on pourrait 
faire courir une narration cohérente de 1991 au moins jusqu'à aujourd'hui principalement, à présent, en territoires serbe, monténégrin et macédonien).

4 Une seconde façon de distinguer ces travaux consiste à tracer une ligne de partage qui concerne la zone potentielle d'exposition du travail, ou encore, pourrait-on dire un peu comme on le fait pour les journaux, sa «zone de diffusion». On peut ainsi distinguer, d'une part, les travaux exposés dans l'espace public français (travaux d'auteurs français ou d'auteurs étrangers francophones, travaux traduits en langue française) et, d'autre part, les travaux présentés dans des espaces publics autres (espaces publics britannique, allemand, anglo-américain, hispano-américain, etc.).

Un troisième critère est celui du point de vue adopté par l'auteur, point de vue qui peut être plus ou moins explicitement mis en valeur ou revendiqué, mais qui existe dans tous les cas, et qui est conditionné par une épistémologie et par des appareillages théoriques et de méthode. On pourra ainsi distinguer le point de vue savant, dit également scientifique (traités, analyses de corpus, investigations diverses soumises à protocole d'enquête...), le point de vue de l'acteur critique, c'est-à-dire le point de vue d'une instance individuelle ou collective interne aux métiers de l'information (on trouvera ici des témoignages de journalistes ${ }^{1}$, des réflexions de professionnels de l'information ${ }^{2}$, etc.), et le point de vue de l'observateur critique, c'est-à-dire le point de vue d'une instance externe aux métiers de l'information. Prend place, dans cette catégorie, tout travail d'un observateur des médias et de leurs productions, quel que soit le degré et l'orientation partisane de cet observateur : du pamphlet d'un défenseur de la cause nationaliste serbe dénonçant le parti pris des médias européens dans leur couverture du conflit ${ }^{3}$, jusqu'à un atelier de réflexion sur l'emploi des mots par la presse française monté par un collectif de citoyens favorables à une Bosnie unitaire ${ }^{4}$, en passant par différents écrits d'essayistes, de personnalités associées à la sphère littéraire ou artistique, etc.

6 L'objet étudié représente un quatrième critère possible. On peut distinguer les travaux qui se réfèrent aux médias de l'espace yougoslave et ceux qui s'intéressent aux médias non-yougoslaves, français notamment ${ }^{5}$. Dans l'un comme dans l'autre cas, les travaux peuvent porter sur l'«amont », c'est-à-dire sur les pratiques professionnelles et les modalités de production de l'information, ou bien sur le "résultat », le "produit fini », c'est-à-dire les formes et les contenus de l'information telle qu'elle est délivrée au public. À l'intérieur de cette seconde catégorie, on peut affiner la distinction selon la nature du matériel étudié (matériel constitué d'énoncés verbaux, ou constitué d'images fixes ou animées). Ces critères typologiques étant posés, nous proposons dans les pages qui suivent une analyse des travaux existants, en laissant néanmoins de côté les études exposées en dehors de l'espace public français ${ }^{6}$.

\section{Tendances et contrastes}

7 Premier constat: comme c'est le cas depuis le commencement des analyses des discours médiatiques, la radio est très clairement négligée. On observe, en revanche, une place relative faite d'une part à la télévision (analysée dans sa dimension verbale et/ou iconique $^{7}$ ) et, d'autre part, aux images fixes ou animées (affiches, photos de presse, livres de photos...8). Malgré cela, dans l'ensemble, la presse écrite reste le support privilégié des travaux, que ceux-ci soient menés depuis une posture savante ${ }^{9}$ ou dans la perspective de l'observateur critique ${ }^{10}$. 
8 Deuxième constat : comme c'est le plus souvent le cas, les analyses de “ produits finis " sont bien plus nombreuses que les analyses de la production - si ce n'est dans les témoignages de journalistes où le sujet (sinon son analyse du moins son illustration) domine, ainsi qu'on peut normalement s'y attendre. Dans ce contexte, une réalisation telle que le documentaire de Marcel Ophuls, Veillées d'armes. Une histoire du journalisme en temps de guerre, sorti en salles en novembre 1994, mérite d'autant plus d'être remarquée. Sur un tout autre mode, les recherches originales de Michael Palmer ${ }^{11}$ sur le travail des agences mondiales d'information, telles que Reuter, apportent également leur contribution à la connaissance des modalités de production de l'information sur les guerres yougoslaves, bien qu'il s'agisse avant tout de l'étude savante d'une métaanalyse professionnelle, et non pas d'une étude en observation de la production des nouvelles. Michael Palmer s'intéresse, en effet, aux évaluations que les journalistes d'agence rédigent dans le cadre de leur travail à propos de la production, du traitement et de la diffusion des dépêches concernant l'espace yougoslave.

9 Cependant, quand on observe l'ensemble des travaux produits, le constat le moins attendu que l'on fait concerne les guerres et périodes étudiées. Par comparaison aux guerres de Slovénie, de Croatie et de Bosnie, la guerre du Kosovo est marquée par les thématiques qu'elle suscite : celles de la communication politique et de la manipulation de l'opinion.

10 À propos de la guerre en Bosnie ${ }^{12}$, certains auteurs s'intéressent aux discours - énoncés verbaux et images - des médias occidentaux pour en proposer l'analyse. C'est principalement ce motif qui habite la majorité des articles publiés dans une livraison de la revue Mots parue en juin 1996 sur le thème: "Les médias dans le conflit yougoslave». C'est également cet objet qui a constitué nos propres recherches ${ }^{13}$. Mais plus nombreux sont les auteurs qui, durant cette même période, se penchent sur les médias appartenant à l'espace yougoslave. Ces travaux, on peut le noter, concluent tous à un rôle déterminant des médias ex-yougoslaves dans l'exacerbation des sentiments nationalistes de tous bords. Sans aucunement dégager les hommes et femmes politiques de leurs responsabilités, ces analyses font peser une lourde charge sur les journalistes yougoslaves et sur les dirigeants d'entreprises médiatiques, que ces travaux soient des enquêtes institutionnelles ${ }^{14}$, des enquêtes menées par des universitaires ${ }^{15}$ ou par des journalistes ${ }^{16}$, ou encore des témoignages de journalistes yougoslaves, tel que celui de l'ancien rédacteur en chef de TV Sarajevo, Nenad Pejic ${ }^{17}$. Bref, dans la période 1991-1995 des guerres yougolaves, on s'intéresse beaucoup aux médias de "l'Autre yougoslave ", aux supports de diffusion des thèses nationalistes (serbes, mais aussi croates voire bosniaques), aux rapports de dépendance entre médias et pouvoirs politiques nationalistes en place $^{18}$.

11 À propos de la guerre du Kosovo qui s'étend de la fin février 1998 à la mi-juin 1999 (avec l'entrée en guerre de l'Otan à partir du 24 mars 1999), les auteurs portent un intérêt prononcé au thème de la construction de l'opinion dans les pays occidentaux et à celui de la communication de l'Otan et des gouvernements nationaux des pays «alliés ». Ce sont ces préoccupations qui motivent la publication, fin 1999, d'un numéro des Cahiers de médiologie intitulé «Croyances en guerre. L'effet Kosovo ». Du côté des travaux à caractère scientifique, la recherche d'Éric Maigret (1999) comme celle de Francis Chateauraynaud (2000) accordent une attention centrale aux stratégies de communication de l'Otan. La perspective de l'observateur critique, représentée par «L'opinion, ça se travaille... » de Serge Halimi et Dominique Vidal (2000) ou par Bombes et 
bobards de David Mathieu (2000), est tout entière marquée par la conviction que l'opinion a été manipulée par une presse et une radio-télévision prises au piège de la communication des pouvoirs politiques et, surtout, militaro-industriels ${ }^{19}$. La période est ainsi marquée par un intérêt pour les médias "alliés ", «occidentaux » - et non plus yougoslaves -, et par la façon dont les pouvoirs politiques et militaires instrumentalisent ces médias dans le but d'influencer l'opinion.

Ces préoccupations qui marquent la seconde période des guerres yougoslaves ne sont pas sans rappeler celles qui suivirent la guerre du Golfe de l'hiver 1990-1991: les commentaires sur les visées manipulatrices des conférences de presse de Jamie Shea, porte-parole de l'Otan durant la guerre du Kosovo, semblent faire suite aux commentaires sur les visées manipulatrices des "pools de presse " organisés par l'armée américaine lors de la guerre du Golfe (de même que les commentaires sur les dangers pour les soldats de l'Onu de l'utilisation par l'otan d'armes à l'uranium appauvri ont semblé faire suite aux commentaires sur l'intoxication des soldats américains par leur propre matériel dans le désert saoudien). De ce point de vue, en termes de thématique et de type de questionnements, il n'y aurait pas linéarité ou progrès sur la trajectoire des trois guerres - Golfe, Bosnie, Kosovo -, mais plutôt un retour en boucle, les thématiques suscitées par la troisième guerre revenant sur celles que suggéra la première, dix ans plus tôt.

\section{Pour conclure : la construction de l'opinion au centre des préoccupations}

L'observation du contraste entre les écrits amenés par la guerre de Bosnie et ceux que provoque la guerre du Kosovo conduit à s'interroger sur le fait de savoir si, de façon plus ou moins consciente, ce n'est pas avant tout la question de la manipulation et de l'influence qui motive les travaux, qu'ils soient savants ou non. À propos des années 1991-1995, soumettant à l'investigation les médias yougoslaves, les auteurs se demandent comment l'opinion yougoslave a pu accepter la guerre, être amenée à prendre les armes ou à soutenir un conflit sanglant mené en son nom. Se référant à la période 1998-1999, se penchant sur le fonctionnement des médias «alliés " dans la communication politique et militaire occidentale, les auteurs se demandent comment l'opinion européenne a pu accepter - ou non - des bombardements meurtriers et destructeurs menés en son nom.

Dans le contexte de telles préoccupations, les travaux sur la réception - au sens où l'on distingue habituellement "production" / «produit» / "réception» - apparaissent encore une fois comme les grands absents de la recherche : si la question de l'influence mobilise en définitive si fortement chercheurs, acteurs et témoins, comment ne pas s'étonner, en effet, du peu d'études mises en œuvre dans ce domaine?

Actes des Assises nationales des collectifs contre la purification ethnique, 1995. Assises tenues à Montreuil les 19 et 20 novembre 1994. Brochure publiée en avril 1995 par le Comité Citoyens-citoyennes pour la Bosnie-Herzégovine.

16 Arakon M., 2001, "La perception des médias turcs dans les guerres du Golfe et du Kosovo ", pp. 315-327, in: Mathien M., dir., L'information dans les conflits armés. Du Golfe au Kosovo, Paris, Éd. L'Harmattan, coll. Communication. 


$$
\text { crise yougoslave », Mots. Les langages du politique (Paris, Presses de Sciences Po), 47, }
$$
pp. 7-22. des troupes serbes pendant la guerre du Kosovo», pp. 429-446, in : M. Mathien, dir. L'information dans les conflits armés. Du Golfe au Kosovo, Paris, Éd. L'Harmattan, coll. Communication.

Charaudeau P., Lochard G. et Soulages J.-C., 1996, «La construction thématique du conflit en ex-Yougoslavie par les journaux télévisés français (1990-1994)», Mots. Les langages du politique (Paris, Presses de Sciences Po), pp. 89-108.

Charaudeau P. et al., 2001, La télévision et la guerre. Déformation ou construction de la réalité ? Le conflit en Bosnie (1990-1994), Paris, INA/De Boeck Université, coll. Médias Recherches.

Chateauraynaud F., 2000, «Les ressorts de la critique à l'échelle internationale. Une lecture pragmatique de quelques épisodes de la guerre du Kosovo", colloque L'historicité de l'action publique, organisé par le Curapp (UMR 6054) et le Groupe de sociologie politique et morale (EHESS), Amiens, Université de Picardie, 12-13 octobre 2000. Actes à paraître aux Presses Universitaires de France.

Chomsky N., 2000, Le Nouvel Humanisme militaire. Leçons du Kosovo, Lausanne, Éditions Page deux, coll. Cahiers libres.

Clarinard R., et Collette J., 2000, Kosovo: les batailles de l'information, Paris, Éd. L'Harmattan, coll. Des hommes et des conflits.

\section{Mathien M., dir., 2001, L'information
L'Harmattan, coll. Communication}



8 d'études et de recherches interdisciplinaires sur les médias en Europe (Cerime), Strasbourg, 23-24 mars 2000. manipulations de la guerre du Kosovo, 24 mars - 10 juin 1999, Éd. L'Âge d'homme, coll. Objections.

\section{l'Otan », pp. 241-257, in : Mathien M., dir., L'information dans les conflits armés. Du Golfe au} Kosovo, Paris, Éd. L'Harmattan, coll. Communication.

Humblot C., 1993, "Les médias fauteurs de guerre ", enquête publiée en plusieurs parties dans le quotidien Le Monde des 22, 23, 24 et 26 juillet 1993.

"Les médias dans le conflit yougoslave», 1996, Mots. Les langages du politique (Paris, Presses de Sciences Po), 47.

Klingelschmitt R., 2001, «Le conflit du Kosovo : les bilans dans les médias allemands", pp. 277-290, in : Mathien M., dir., L'information dans les conflits armés. Du Golfe au Kosovo, Paris, Éd. L'Harmattan, coll. Communication. pp. 109-126. langage, Université Paris 13, 3 volumes.

le nom des camps découverts en Bosnie ", Langage et Société (Paris, Maison des Sciences de l'Homme), 93, pp. 33-69.

Les Cahiers de médiologie, 1999, «Croyances en guerre. L'effet Kosovo » (Paris, Gallimard), nationalismes ", Études (Assas-Editions, Paris), tome 378, 4, pp. 437-447.

Lutard C., 1993b, «Dérive autoritaire en Croatie et en Serbie », Le Monde diplomatique (Paris), p. 14.

Maigret É., 1999, «Le journal télévisé de Bagdad à Belgrade. Le problème du récit par temps de guerre transnationale ", in: Fleury-Vilatte B., dir., Récit médiatique et histoire, Paris, Éd. L'Harmattan/Ina, à paraître (actes du colloque Récit médiatique et histoire, Nancy, 5-6 novembre 1999).

Mathien M., dir., 2001, L'information dans les conflits armés. Du Golfe au Kosovo, Paris, Éd. L'Harmattan, coll. Communication, 526 p. Actes du colloque organisé par le Centre Mathieu D., 2000, Bombes et bobards. Propagande, bourrage de crâne, mensonges et

Mots. Les langages du politique, 1996, «Les médias dans le conflit yougoslave » (Paris, Presses de Sciences Po), 47.

Ophuls M., 1994, Veillées d'armes. Une histoire du journalisme en temps de guerre, documentaire long métrage.

Palmer M., 1996, «Agences de presse : urgence et concurrence », Mots. Les langages du politique (Paris, Presses de Sciences Po), 47, pp. 73-88. 
Palmer M., 2001, «L'“apotheose now” ou le discours de Reuters lors de l'intervention de l'Otan », pp. 213-226, in: M. Mathien, dir., L'information dans les conflits armés. Du Golfe au Kosovo, Paris, Éd. L'Harmattan, coll. Communication.

«Paroles de journalistes de l'ex-Yougoslavie », dossier de Hommes et Libertés (revue de la Ligue des Droits de l'Homme, Paris), 74, septembre 1993, pp. 24-33.

Pedon É. et Walter J., 1996, « Les variations du regard sur les “camps de concentration” en Bosnie. Analyse des usages de la photographie dans un échantillon de journaux français ", Mots. Les langages du politique (Paris, Presses de Sciences Po), 47, pp. 23-45.

Pedon É. et Walter J., 2001, "La couverture photographique du conflit du Kosovo. Comparaison des "unes" des quotidiens Libération et Le Monde ", pp. 417-428, in: M. Mathien, dir., L'information dans les conflits armés. Du Golfe au Kosovo, Paris, Éd. L'Harmattan, coll. Communication.

4 Pejic N., 1993, «Les médias sont responsables de la guerre civile en ex-Yougoslavie ”, Médiaspouvoirs (Paris), 29, pp. 25-31.

Regards médiatiques et perspectives nationales sur les conflits guerriers. La guerre en ex-Yougoslavie (1990-1999), journée d'étude organisée par le Laboratoire Communication et Politique du CNRS, Paris, 30 mars 2000.

«Situation des droits de l'homme dans le territoire de l'ex-Yougoslavie. Rapport spécial sur les médias ", 13 décembre 1994. Commission des droits de l'homme. Nations unies. Conseil économique et social, cinquante et unième session. Document E/CN.4/1995/54.

Stoyanne D., 1994, Petit glossaire de la guerre civile yougoslave, numéro double spécial de Raison Garder, Revue de l'Institut Serbe de Lausanne, Nouvelle série, 5-6.

Télérama, 1993, Dossier sur la propagande et les médias yougoslaves, 14 avr., pp. 8-14.

Thompson M., 1994, Forging War: the Media in Serbia, Croatia, Bosna-Hercegovina, Avon, The Bath Press.

Trapouzanlis C., 2001, «Les médias grecs et l'intervention de l'Otan », pp. 307-314, in : Mathien M., dir., L'information dans les conflits armés. Du Golfe au Kosovo, Paris, Éd. L'Harmattan, coll. Communication.

\section{NOTES}

1. Voir par exemple le témoignage du grand reporter de Libération J. Hatzfeld (1994).

2. Tels que certains débats et conférences organisés par l'association Reporters sans frontières sur le thème de la couverture de la guerre par les médias français ou européens.

3. Voir par exemple le livret de D. Stoyanne (1994).

4. Voir par exemple l'atelier « Analyse des concepts et du vocabulaire utilisés par les médias, les politiques, et par nous, à propos de la Bosnie et de la situation en ex-Yougoslavie", dans les Actes des Assises nationales des collectifs contre la purification ethnique (1995 : 23-26).

5. Comme on peut s'y attendre, le domaine français fait la part belle aux médias français. Il importe d'autant plus de remarquer la place accordée à des médias qui ne sont ni français ni yougoslaves lors du colloque organisé par le Cerime (Centre d'études et de recherches 
interdisciplinaires sur les médias en Europe) à Strasbourg en mars 2000 (voir les actes dans M. Mathien, dir., 2001), à travers les interventions de A.C. Montant-Ilhat (2001) pour la Russie, M. Hartmeier (2001) et R. Klingelschmitt (2001) pour l'Allemagne, M. Arakon (2001) pour la Turquie, C. Trapouzanlis (2001) pour la Grèce. À noter également, pour la Roumanie, l'intervention de I. Dragan, «La guerre du Kosovo vue de Roumanie », lors de la journée Regards médiatiques et perspectives nationales sur les conflits guerriers (2000).

6. Signalons tout de même pour mémoire deux ouvrages britanniques importants. L'un comporte des analyses des formes et des contenus télévisuels ainsi que des analyses et témoignages sur les pratiques professionnelles des journalistes étrangers en territoire yougoslave (Gow, Paterson, Preston, dir., 1996). L'autre porte sur le rôle des médias yougoslaves dans les nationalismes et dans la guerre (Thompson, 1994).

7. Voir en particulier: P. Charaudeau et al. (2001). P. Charaudeau, G. Lochard et J.-C. Soulages (1996). P. Charaudeau, «La guerre de Bosnie dans les 20 h00», lors de la journée Regards médiatiques et perspectives nationales sur les conflits guerriers (2000). J.-C. Soulages dans le présent numéro de Questions de communication. Voir également: É. Maigret (1999) et B. Fleury-Vilatte (2001).

8. Voir en particulier : É. Pedon et J. Walter (1996 et 2001), et É. Pedon et J. Walter dans le présent numéro de Questions de communication.

9. Nos propres recherches (Krieg, 1996, 2000a et 2000b) privilégient nettement la presse écrite. Mettent également l'accent sur ce support : S. Bonnafous (1996) et P. Breton (2001).

10. Ainsi, les propositions que le syndicaliste et militant politique belge M. Collon expose dans ses deux ouvrages (Collon, [1992] 1994 et 1998) reposent sur un matériel dont l'essentiel est composé d'articles de presse, même si aucun matériel n'est exclu a priori du commentaire. De même, le livre de S. Halimi et D. Vidal (2000) met en bonne place la presse écrite, même si les auteurs s'intéressent y compris à la dimension iconique de celle-ci (les photos et leurs légendes, dispositifs des Unes et des couvertures de magazines, etc.)

11. M. Palmer (1996). M. Palmer, «Les agences d'information et la guerre du Kosovo », lors de la journée Regards médiatiques et perspectives nationales sur les conflits guerriers (2000), repris dans Palmer (2001).

12. Il est légitime de parler de la période 1991-1995 comme de celle des guerres en Slovénie, Croatie et Bosnie; mais c'est malgré tout le territoire bosniaque qui attire à lui le plus de commentaires et de polémiques.

13. A. Krieg (1996, 2000a et 2000b).

14. Par exemple Situation des droits de l'homme dans le territoire de l'ex-Yougoslavie. Rapport spécial sur les médias, décembre 1994, Nations unies.

15. Par exemple R. de la Brosse (1995) ou C. Lutard (1993a et 1993b).

16. Par exemple C. Humblot (1993) ou encore le dossier de Télérama nº 2257, 14 avril 1993.

17. N. Pejic (1993). Voir également «Paroles de journalistes de l'ex-Yougoslavie», septembre 1993.

18. Dans le même ordre d'idées, resterait à faire l'analyse plus large des supports de propagande, à travers les "produits dérivés" du nationalisme d'une part (cassettes de chansons, bandes dessinées, affichettes, écussons, ou encore portes-clés, tels que les portes-clés bifaces à l'effigie de Milosevic et de Saint-Sava qui circulent à partir de 1989 en Serbie et au Kosovo), et à travers les récits et rumeurs mis en circulation sur le terrain d'autre part.

19. Voir également R. Clarinard et J. Collette (2000) et C. Guillon (2000). On distinguera ce type d'étude, centré prioritairement sur les médias, de celui qui relève plus nettement de l'analyse géopolitique de la guerre. Ainsi par exemple de l'ouvrage de M. Collon (2000) et de celui de N. Chomsky (2000). 


\section{RÉSUMÉS}

Cet article propose un état des lieux des travaux (au sens large : recherches savantes mais aussi témoignages, pamphlets, essais...) qui portent sur le thème "médias et guerres yougoslaves ". Après avoir proposé une typologie des travaux existants, on s'intéresse aux tendances et contrastes que ceux-ci présentent. Il apparaît que la variable la plus discriminante est celle du conflit : alors que les guerres en Croatie et en Bosnie (1991-1995) suscitent nombre de travaux sur le rôle des médias yougoslaves dans l'expression du nationalisme, la guerre du Kosovo (1998-1999) favorise le thème de la communication politique dans les pays occidentaux. Une préoccupation rassemble ces deux séries: l'influence des médias dans la construction de l'opinion publique.

\section{INDEX}

Mots-clés : guerres dans l'ex-Yougoslavie (1991-1999), médias, communication politique, construction de l'opinion

\section{AUTEUR}

\section{ALICE KRIEG}

Centre d'étude des discours, images, textes, écrits, communications

Université Paris 12

akrieg@club-internet.fr 